Modelling late stool frequency and rectal pain after radical radiotherapy in prostate cancer patients: Results from a large pooled population

A. Cicchetti 1, T. Rancati 1, M. Ebert 2,3 C. Fiorino 4, F. Palorini 1, A. Kennedy 3, D.J. Joseph 3, J.W. Denham 5, V. Vavassori 6, G. Fellin 7, R. Valdagni 1,8,9

1 Prostate Cancer Program, Fondazione IRCCS Istituto Nazionale dei Tumori, Milan, Italy 2 Medical Physics, University of Western Australia, Perth, Western Australia

3 Physics Research, Sir Charles Gairdner Hospital, Perth, Western Australia

4 Medical Physics, San Raffaele Scientific Institute, Milan, Italy

5 School of Medicine and Public Health, University of Newcastle, New South Wales, Australia

6 Radiotherapy, Cliniche Humanitas-Gavazzeni, Bergamo, Italy

7 Radiotherapy, Ospedale Santa Chiara, Trento, Italy

8 Oncology and Hemato-oncology, Università degli Studi di Milano, Milan, Italy

9 Radiation oncology 1, Fondazione IRCCS Istituto Nazionale dei Tumori, Milan, Italy

Corresponding author:

Alessandro Cicchetti

Prostate Cancer Program

Fondazione IRCCS Istituto Nazionale dei Tumori

Via Venezian 1 - 20133 Milano, Italy

+390223903009

Alessandro.cicchetti@istitutotumori.mi.it

Conflict of interest statement: authors have nothing to disclose 


\begin{abstract}
Aim:

To investigate late gastrointestinal toxicity in a large pooled population of prostate cancer patients treated with radical radiotherapy. Normal tissue complication probability models were developed for late stool frequency and late rectal pain.
\end{abstract}

\title{
Methods and materials:
}

Population included 1336 patients with 3-year minimum follow-up, treated with 66-80Gy. Toxicity was scored with LENT-SOMA scale. Two toxicity endpoints were considered: grade $\geq 2$ rectal pain and mean grade (average score during follow-up) in stool frequency $>1$.

DVHs of anorectum were reduced to equivalent uniform dose (EUD). The best-value of the volume parameter $\mathrm{n}$ was determined through a sensitivity analysis. Association between EUD/clinical factors and the endpoints was investigated by logistic analyses. Likelihood and calibration were used to evaluate models.

Results:

$4 \%$ of patients (45/1122) reported mean stool frequency grade $>1$; grade $\geq 2$ rectal pain was present in the TROG 03.04 RADAR population only (21/677, 3.1\%): for this endpoint, the analysis was limited to this population.

Analysis of DVHs highlighted the importance of mid-range doses (30-50Gy) for both endpoints.

EUDs calculated with $n=1(O R=1.04)$ and $n=0.35(O R=1.06)$ were the most suitable dosimetric descriptors for stool frequency and rectal pain respectively.

The final models included EUD and cardiovascular diseases $(\mathrm{OR}=1.78)$ for stool frequency and EUD and presence of acute gastrointestinal toxicity $(\mathrm{OR}=4.2)$ for rectal pain.

\section{Conclusion:}

Best predictors of stool frequency and rectal pain are consistent with findings previously reported for late fecal incontinence, indicating an important role in optimization of mid-range dose region to minimize these symptoms highly impacting the quality-of-life of long surviving patients.

Key words: radiotherapy, prostate cancer, late toxicity, dose-volume models 


\section{INTRODUCTION}

The majority of studies devoted to gastrointestinal side effects after radiotherapy for prostate cancer are focused on rectal bleeding, faecal incontinence and overall acute gastrointestinal toxicity. These studies often resulted in the quantification of dose-volume effects for organs-at-risk (primary the rectum) and led to the development of predictive models for radiotherapy-induced toxicity, which sometimes including clinical risk factors [1-3].

Nevertheless, chronic radioinduced rectal syndrome includes other symptoms [4], such as urgency, increased stool frequency and rectal pain. There is insufficient knowledge on the incidence of these morbidities and on their relationship with the dose distribution in the rectum and in the anal canal. This is mainly due to their being relatively rare effects; there is difficulty in identifying radiation as the cause of these impairments in an ageing population and the lack of controlled questionnaire-based prospective scoring describing the pre-radiotherapy baseline situation.

Nevertheless, these symptoms may clearly have a non-negligible impact on the quality of life (QoL) of long-surviving patients [5] and consequently deserve attention.

The availability of a large dataset consisting of a pooled population from two large prospective trials $[6,7]$ that represents more than one thousand patients with a minimum of 3 years of follow-up allowed us to focus on these often neglected gastrointestinal side effects: stool frequency and rectal pain.

The two cohorts were treated at different hospitals, with different dose levels, with different radiotherapy techniques, in different countries and in different time frames. As a consequence, current pooled population presented with a wide variety of dosimetric and clinical parameters, with the potential to reach sufficient statistical power to assess main associations between the selected rare side effects and clinical/dosimetric features. The aim of current study was to develop multivariable logistic (MVL) regression models for both the above mentioned toxicity endpoints. 


\section{MATERIALS and METHODS}

\section{Patient population}

A pooled population from two high quality multicentre prospective trials on radiotherapy for prostate cancer was created.

a. Airopros 0102: a prospective multicentre observational trial specifically designed to evaluate dosimetric/clinical factors associated with acute and late rectal syndrome symptoms after radical radiotherapy for prostate cancer (details can be found in [6,8-10])

b) TROG03.04 RADAR: a prospective multicentre randomized trial designed to determine whether adjuvant androgen suppression, bisphosphonates and radiation dose escalation might improve oncologic outcomes in localized prostate cancer (details in [7,11-15]). In this trial, $d$ toxicity was prospectively scored as a secondary endpoint.

All patients were treated with radical three-dimensional conformal radiotherapy (3DCRT) in the period 2002-2004 and 2004-2007 for Airopros 0102 and TROG03.04 RADAR, respectively. The prescribed dose was between 66 and 80 Gy (median 73.2 Gy, interquartile range 70-75 Gy, 1.8-2 Gy/day in all cases). A constraint of 70 Gy to the rectum was applied to the RADAR population.

Co-morbidities (diabetes, hypertension, cardiovascular diseases, and the presence of haemorrhoids), previous abdominal/pelvic surgery, use of drugs and previous/concomitant loco-regional diseases were evaluated with a specifically designed questionnaire administered prior to radiotherapy. Information on the quality and duration of the hormonal therapy was also recorded when prescribed to the patient. These clinical information was collected from both trial in a similar way.

Other information concerning the volume definition, planning and treatment modalities as well as the distribution of the main clinical parameters of the two populations have previously been reported in detail $[6-8,11]$. Of particular importance is the delineation of the anorectum: an anatomically based definition (from the anus to the point where the rectum turns into the sigmoid) was used by the participating centres in both trials. This definition was previously found to be sufficiently robust for the aims of dose-volume studies. The dose-volume histogram (DVH) of the solid rectum was considered.

$\underline{\text { Toxicity assessment and endpoint definition }}$ 
Patients were assessed at the start and the end of radiotherapy and every 6 months thereafter for at least 3 years of follow-up.

Gastrointestinal symptoms were determined according to the LENT/SOMA (Late Effects of Normal Tissue/Subjective, Objective, Management and Analytic) scoring systems for late radiation morbidity. Late rectal pain and stool frequency were analysed in the current study.

Rectal pain was considered as a peak toxicity and grade $\geq 2$ (i.e., intermittent $\&$ tolerable OR persistent $\&$ intense pain) was scored as a toxicity event. We defined a late event if it occurred in the time frame 6-36 months after the end of 3DCRT. For stool frequency, we chose a longitudinal definition; mean stool frequency was defined as the average score during the 3-year period after RT. Patients with at least three out of six follow-up points were included in this analysis. An average mean stool frequency $>1$ was arbitrarily considered as the endpoint as it selects those patients with persistent symptoms (i.e., patients who on average evacuated $>2$ times/day), more likely to be those whose symptoms are actually due to radiotherapy.

Longitudinal definitions of toxicity were already considered for faecal incontinence by Gulliford et al. [16] and Fiorino et al. [17], which suggested that the longitudinal approach is more appropriate in describing both the severity and persistence of moderate symptoms that are very important for QoL and the social activities of patients.

The baseline questionnaire was used to exclude patients with symptoms that were already present before radiotherapy, while the end of treatment evaluation was defined acute gastrointestinal toxicity following the RTOG/EORTC definition.

\section{Development of multivariable logistic models}

Univariable logistic regression was used to determine the association between toxicity and clinical/dosimetric/treatment related factors.

The rectal dose-volume histogram (DVH) was reduced to the Equivalent Uniform Dose (EUD).

The value of the rectum volume parameter $\mathrm{n}$ for the two considered toxicity endpoints was determined through a sensitivity analysis: a set of EUDs were computed for a set of $n$-values ranging from 0 to 1 in 0.05 steps. These EUDs were inserted in univariate logistic models and n-value maximizing log likelihood (LLH) was chosen as the most suitable.

The MVL analysis was performed to include all covariates that were to be associated with the endpoint in 
the univariate analysis (covariates with $\mathrm{p}<0.15)$. The odds ratio $(\mathrm{OR})$ was used to express the strength of association of a parameter with the considered symptom.

The goodness-of-fit was determined through the Hosmer-Lemeshow (HL) test and the calibration plot (slope coefficient and $\mathrm{R}^{2}$ ).

All statistical analyses were performed using KNIME software (KNIME GmbH, Germany) coupled with R software (www.r-project.org).

\section{RESULTS}

\section{$\underline{\text { Patient characteristics }}$}

The merged dataset consisted of 1337 patients. A total of 1122 (445 patients from Airopros0102 and 677 from TROG 03.04 RADAR) with complete clinical and dosimetric information and a minimum follow up of 3 years were considered for analysis.

Table 1 shows the characteristics of the patients included in the analysis. The two populations were significantly different for most clinical/treatment related parameters. Notably, all TROG 03.04 RADAR patients received neoadjuvant/adjuvant hormone therapy and irradiation of seminal vesicles, whereas irradiation of the pelvic nodes was not allowed in this trial.

1122 patients were included in the analysis of stool frequency (i.e., patients with no baseline symptoms and with at least 3 follow-up points in 3 years), while 677 were handled for rectal pain.

Toxicity rates were as follows:

1. Mean stool frequency $>1$ : 4\% (45/1122 patients, 5.4\% in the TROG 03.04 RADAR population and $2.2 \%$ in Airopros 0102).

2. Grade $\geq 2$ rectal pain: $2.2 \%$ (21/677 patients, $3.8 \%$ in the TROG 03.04 RADAR population and $0 \%$ in the Airopros 0102)

Due to absence of rectal pain events in the Airopros 0102 population, analysis of this endpoint was limited to the TROG 03.04 RADAR population.

Determination of the volume parameter $\mathrm{n}$ 
Figure 1 reports the mean DVHs (together with standard deviation and p-values from the t-test for the different DVH cut-off points) for patients with and without toxicity for both endpoints.

EUD was calculated for n-values between 0 and 1 in 0.05 steps. Figure 2 (a and b) depicts the EUD values as a function of the volume parameter $\mathrm{n}$ for patients with and without the selected toxicity endpoints. Figure 2 ( $\mathrm{c}$ and d) shows LLH for the univariate logistic model as a function of $\mathrm{n}$. LLH was maximized for $n=1$ (i.e., EUD is the mean rectal dose) for mean stool frequency $>1$ and for $n=0.35$ for late rectal pain.

\section{Multivariable model for mean stool frequency $>1$}

Details on univariate analysis including clinical risk factors are reported in the supplementary materials. Mean stool frequency was associated with the presence of cardiovascular diseases and the development of grade $\geq 2$ acute gastrointestinal toxicity. A MVL resulted with the inclusion of cardiovascular diseases $(\mathrm{OR}=1.78, \mathrm{p}=0.09)$ and EUD calculated with $\mathrm{n}=1(\mathrm{OR}=1.04, \mathrm{p}=0.07)$.

The calibration plot (presented in the supplementary material) had a slope $=1.03\left(R^{2}=0.94\right)$, whereas the p-value for the HL test was 0.88; the LLH was -198.6.

Adding grade $\geq 2$ acute gastrointestinal toxicity to the two variable model did not improve fitting $\left(\mathrm{LLH}=-199.1\right.$, calibration slope $=1.01, \mathrm{R}^{2}=0.94$, $\mathrm{p}$-value for HL test $\left.=0.82\right)$.

Figure 3 shows the probability of mean stool frequency $>1$ as a function of EUD and the presence of cardiovascular diseases. Observed toxicity rates are reported together with model curves.

\section{$\underline{\text { Multivariable model for late rectal pain grade } \geq 2}$}

As explained before, for modelling of late rectal pain, only the TROG 03.04 RADAR population was considered (677 patients, 21/677 toxicity events).

The presence of hormone therapy and irradiation of seminal vesicles and lymph nodes could not be considered as covariates in this population due to the previously explained homogeneity of the patients with respect to these factors.

Details on the univariate analysis including clinical risk factors are reported in the supplementary materials. 
Presence of grade $\geq 2$ acute toxicity was the only clinical factor found to be significantly associated with late rectal pain at the univariate level. Even if a model including acute toxicity cannot be considered as a pre-radiotherapy predictive model, we chose to insert this highly explicative variable in a MVL regression. This could provide guidance during early follow up of patients by indicating those at higher risk of having moderate/severe late rectal pain.

The final two variable models included grade $\geq 2$ acute gastrointestinal toxicity $(\mathrm{OR}=4.2, \mathrm{p}<0.01)$ and EUD calculated with $\mathrm{n}=0.35(\mathrm{OR}=1.06, \mathrm{p}=0.12)$.

The calibration plot (presented in supplementary material) had a slope $=0.98\left(\mathrm{R}^{2}=0.63\right)$, whereas the p-value for HL test was 0.47. LLH was -89 .

Inclusion of acute toxicity slightly improved the model in goodness-of-fit with respect to the one only including EUD (calibration slope $=0.96, \mathrm{R}^{2}=0.88$ and $\mathrm{p}$-value for HL test=0.65, LLH=-88.9).

Figure 4 shows the probability of late rectal pain grade $\geq 2$ as a function of EUD and the presence of grade $\geq 2$ acute gastrointestinal toxicity. Observed toxicity rates are reported together with model curves.

\section{DISCUSSION}

The current scientific knowledge considers radioinduced side effects as being associated with a large number of clinical/dosimetric factors that differ for each individual patient. Inferring these multifaceted models, including dosimetric and clinical features, directly from the current mechanistic radiobiological understanding is not a feasible approach, due to the intrinsically too complex nature of the involved mechanisms. For this reason, models developed in recent years have been derived from the exploration of clinical observations of large prospectively collected populations. These data-driven models aim to provide a phenomenological description of a complex situation and give indications of aspects that could be optimized to limit toxicity in future patients [18].

The availability of a large pooled population of patients treated with radical radiotherapy for prostate cancer, objective evaluation of baseline function and a long prospective follow-up allowed analysis of less common gastrointestinal symptoms (stool frequency and rectal pain) and the development of data-driven models for these types of toxicities. 
Late stool frequency was described using a longitudinal definition (mean frequency $>1$ in the first 3 years after the end of radiotherapy. This choice is crucial as endpoints based on peak toxicity have a high probability to be associated to causes other than radiotherapy, as previously discussed [17].

This toxicity was found to be associated with mean rectal dose (EUD calculated with $n=1)$ ) and the presence of cardiovascular disease $(\mathrm{OR}=1.78)$. Previously published results on this toxicity endpoint are sparse and include slightly different definitions/organs at risk than the present work. Nevertheless there is general agreement on the importance of mean rectal dose/large volumes included in the medium range of doses (30-50 Gy, see figure 1). In a previously published analysis on the TROG 03.04 RADAR population, Ebert et al [12] found significant association between stool frequency defined as peak toxicity and the mid-to-low dose range (8-58 Gy) in the anal canal and with mid-to-high doses in the anorectum. Defraene et al [19] presented a model for incidence of severe frequency ( $>6$ bowel movements/day) including mean dose, while Schaake et al. [20] highlighted the association between anorectal side effects and different anatomical substructures within and around the rectum. Toxicity scoring was in this case based on the CTCAE scale (defined as incidence and not longitudinally). They found that stool frequency was significantly associated with the volume of the levator ani receiving more than $40 \mathrm{~Gy}$ and with the volume of coccygeal muscle irradiated at more than $45 \mathrm{~Gy}$. The significant volume parameters for EUD calculation were in the range $0.5-1$.

When considering clinical factors acting as dose-response modifiers, cardiovascular diseases were already found to be a risk factor for enhanced toxicity for a number of other late gastrointestinal endpoints by previously published trials [19,21]. Patients harbouring these types of comorbidities should be granted special attention, optimization and/or prophylactic treatment.

Late rectal pain was found to be associated with rectal volumes receiving 30-50 Gy (see Figure 1) and with an EUD calculated with $n=0.35$. Patients exhibiting grade $\geq 2$ acute gastrointestinal toxicity were at a higher risk of late moderate/severe pain $(\mathrm{OR}=4.2)$. This could be related to a consequential effect between acute injury and late pain and to a possibly enhanced radiosensitivity of some patients who report worse acute injury and more severe late toxicity. Patients showing intense acute reactions might be require a stricter follow up for sudden treatment and mitigation of this type of morbidity. A previous analysis of the TROG 03.04 RADAR population referred to rectal pain of any grade [12] was not able to find a dose-response relationship. The inclusion of grade 1 pain (occasional \& mild pain) probably blurred the relationship between this endpoint and doses. 
The recent work by Schaake et al. [20] was the only other study that considered the rectal pain endpoint, but they were not able to find any associations between pain and dosimetric/clinical factors.

Findings from analysis of stool frequency and rectal pain are coherent with what was already found in a number of studies when modelling late fecal incontinence $[16,17,19]$. For all these symptoms there is a relevant indication on the role of mid-range doses to large volumes of the anorectum, which is markedly different from the dose-volume relationship for rectal bleeding, which is related to low volumes receiving high doses. This parallel-like behavior of the anorectum could be biologically related to radioinduced fibrosis and consequent rectal stiffness due to large volumes receiving doses around 30-50 Gy. An interesting study on investigation of the patophysiology of anorectal radioinduced toxicity considered the relationship between anal/rectum pressures, rectal capacity and sensory functions and side effects [22,23]. They found that radioinduced rectal stiffness seems associated with rectal wall dysfunction and toxicity.

\section{CONCLUSIONS}

Two models were developed to describe the relationship between stool frequency/rectal pain and dosimetric/clinical risk factors.

In a large pooled population that included more than 1000 patients from two prospective trials, the mean rectal dose was found to be associated persistently increased stool frequency in the first three years after radiotherapy.

Large volumes of the rectum irradiated to mid-range doses ( 30 to $50 \mathrm{~Gy}$ ) were found to be related to both stool frequency and rectal pain (for this latter endpoint, EUD calculated with the volume parameter $\mathrm{n}=0.35$ gave the best dose-response relationship).

Cardiovascular diseases and acute gastrointestinal toxicity were significant risk factors the two symptoms.

All of these findings are coherent with previous modelling of late faecal incontinence [16,17,19], suggesting that the sparing of the ano-rectum from low-to-mid-range doses may reduce the incidence of these symptoms, having a high impact on the QoL of long-surviving patients.

Use of intensity modulated radiotherapy and of stereotactic techniques, with their possibility of reducing low-to-mid doses over large volumes, has the potential to avoid these toxicities. 


\section{AKNOLEDGEMENTS}

The study was funded by: AIRC IG16087, Fondazione Monzino, NHMRC (300705, 455521, and 1006447)

\section{REFERENCES}

1. Fiorino C, Valdagni R, Rancati T, Sanguineti G. Dose-volume effects for normal tissues in external radiotherapy: pelvis. Radiother Oncol 2009;93:153-67.

2. Michalski JM, Gay H, Jackson A, Tucker SL, Deasy JO. Radiation Dose-Volume Effects in Radiation-Induced Rectal Injury. Int J Radiat Oncol Biol Phys 2010;76:S123-9.

3. Landoni V, Fiorino C, Cozzarini C, Sanguineti G, Valdagni R, Rancati T. Predicting toxicity in radiotherapy for prostate cancer. Phys Med. 2016 Mar;32(3):521-32. doi: 10.1016/j.ejmp.2016.03.003.

4. Denham JW, O'Brien PC, Dunstan RH, Johansen J, See A, Hamilton CS et al. Is there more than one late radiation proctitis syndrome? Radiother Oncol. 1999 Apr;51(1):43-53.

5. Krol R, Smeenk RJ, van Lin EN, Hopman WP. Impact of late anorectal dysfunction on quality of life after pelvic radiotherapy. Int J Colorectal Dis. 2013 Apr;28(4):519-26. doi: 10.1007/s00384-012-1593-5.

6. Fellin G, Fiorino C, Rancati T, Vavassori V, Baccolini M, Bianchi C et al. Clinical and dosimetric predictors of late rectal toxicity after conformal radiation for localized prostate cancer: results of a large multicenter observational study. Radiother Oncol. 2009 Nov;93(2):197-202. doi: 10.1016/j.radonc.2009.09.004.

7. TROG. TROG clinical trials summary. TROG 03.04 - randomised trial investigating the effect on survival and PSA control of different durations of adjuvant androgen deprivation in association with definitive radiation treatment for localised carcinoma of the prostate (RADAR) TROG Clinical Trials Summary: TROG, 2005.

8. Valdagni R, Kattan MW, Rancati T, Yu C, Vavassori V, Fellin G et al. Is it time to tailor the prediction of radio-induced toxicity in prostate cancer patients? Building the first set of nomograms for late rectal syndrome. Int J Radiat Oncol Biol Phys. 2012 Apr 1;82(5):1957-66. doi: 10.1016/j.ijrobp.2011.03.028.

9. Fellin G, Rancati T, Fiorino C, Vavassori V, Antognoni P, Baccolini M et al. Long term rectal function after high-dose prostatecancer radiotherapy: results from a prospective cohort study. 
Radiother Oncol. 2014 Feb;110(2):272-7. doi: 10.1016/j.radonc.2013.09.028.

10. Rancati T, Fiorino C, Fellin G, Vavassori V, Cagna E, Casanova Borca V et al. Inclusion of clinical risk factors into NTCP modelling of late rectal toxicity after high dose radiotherapy for prostate cancer. Radiother Oncol. 2011 Jul;100(1):124-30. doi: 10.1016/j.radonc.2011.06.032.

11. Denham JW1, Wilcox C, Lamb DS, Spry NA, Duchesne G, Atkinson C et al. Rectal and urinary dysfunction in the TROG 03.04 RADAR trial for locally advanced prostate cancer. Radiother Oncol. 2012 Nov;105(2):184-92. doi: 10.1016/j.radonc.2012.09.018.

12. Ebert MA, Foo K, Haworth A, Gulliford SL, Kennedy A, Joseph DJ, Denham JW. Gastrointestinal dose-histogram effects in the context of dose-volume-constrained prostate radiation therapy: analysis of data from the RADAR prostate radiation therapy trial. Int J Radiat Oncol Biol Phys. 2015 Mar 1;91(3):595-603. doi: 10.1016/j.ijrobp.2014.11.015.

13. Ebert MA, Harrison KM, Howlett SJ, Cornes D, Bulsara M, Hamilton CS et al. Dosimetric intercomparison for multicenter clinical trials using a patient-based anatomic pelvic phantom. Med Phys. 2011 Sep;38(9):5167-75.

14. Ebert MA1, Haworth A, Kearvell R, Hooton B, Coleman R, Spry N et al. Detailed review and analysis of complex radiotherapy clinical trial planning data: evaluation and initial experience with the SWAN software system. Radiother Oncol. 2008 Feb;86(2):200-10.

15. Kearvell R1, Haworth A, Ebert MA, Murray J, Hooton B, Richardson S et al. Quality improvements in prostate radiotherapy: outcomes and impact of comprehensive quality assurance during the TROG 03.04 'RADAR' trial. J Med Imaging Radiat Oncol. 2013 Apr;57(2):247-57. doi: 10.1111/1754-9485.12025.

16. Gulliford SL1, Partridge M, Sydes MR, Andreyev J, Dearnaley DP. A comparison of dose-volume constraints derived using peak and longitudinal definitions of late rectal toxicity. Radiother Oncol. 2010 Feb;94(2):241-7. doi: 10.1016/j.radonc.2009.12.019.

17. Fiorino C, Rancati T, Fellin G, Vavassori V, Cagna E, Casanova Borca V et al. Late fecal incontinence after high-dose radiotherapy for prostate cancer: better prediction using longitudinal definitions. Int J Radiat Oncol Biol Phys. 2012 May 1;83(1):38-45. doi: 10.1016/j.ijrobp.2011.06.1953.

18. van der Schaaf A, Langendijk JA, Fiorino C, Rancati T. Embracing phenomenological approaches to normal tissue complication probability modeling: a question of method. Int J Radiat Oncol Biol Phys 2015;91:468-71. doi: 10.1016/j.ijrobp.2015.02.059.

19. Defraene G1, Van den Bergh L, Al-Mamgani A, Haustermans K, Heemsbergen W, Van den 
Heuvel F, Lebesque JV. The benefits of including clinical factors in rectal normal tissue complication probability modeling after radiotherapy for prostate cancer. Int J Radiat Oncol Biol Phys. 2012 Mar 1;82(3):1233-42. doi: 10.1016/j.ijrobp.2011.03.056.

20. Schaake W, van der Schaaf A, van Dijk LV, Bongaerts AH, van den Bergh AC, Langendijk JA. Normal tissue complication probability (NTCP) models for late rectal bleeding, stool frequency and fecal incontinence after radiotherapy in prostate cancer patients. Radiother Oncol. 2016 May 3. pii: S0167-8140(16)31039-8.

21. Tucker SL1, Dong L, Bosch WR, Michalski J, Winter K, Mohan R et al. Late rectal toxicity on RTOG 94-06: analysis using a mixture Lyman model. Int J Radiat Oncol Biol Phys. 2010 Nov 15;78(4):1253-60. doi: 10.1016/j.ijrobp.2010.01.069.

22. Krol R, Hopman WP, Smeenk RJ, Van Lin EN. Increased rectal wall stiffness after prostate radiotherapy: relation with fecal urgency. Neurogastroenterol Motil. 2012 Apr;24(4):339-e166. doi: 10.1111/j.1365-2982.2011.01858.x.

23. Krol R1, Smeenk RJ, van Lin EN, Yeoh EE, Hopman WP. Systematic review: anal and rectal changes after radiotherapy for prostate cancer. Int J Colorectal Dis. 2014 Mar;29(3):273-83. doi: 10.1007/s00384-013-1784-8. 


\section{CAPTIONS TO FIGURES}

Figure 1: (A) Relative mean rectal dose-volume histogram (DVH) for patients with/without mean stool frequency $>1$. (B) Relative mean rectal DVH for patients with/without late grade $\geq 2$ late rectal pain. (C) p-values for t-tests for differences in DVHs for patients with/without mean stool frequency $>1$. (D) $\mathrm{p}$-values for t-tests for differences in DVHs for patients with/without late grade $\geq 2$ late rectal pain.

Figure 2: (A) mean equivalent uniform dose (EUD) as a function of volume parameter $\mathrm{n}$ for patients with mean stool frequency $>1$ and with mean stool frequency $\leq 1$. (B) EUD as a function of volume parameter $n$ for patients with grade $\geq 2$ late rectal pain and with grade $<2$ late rectal pain. (C) Log-likelihood (LLH) as a function of $\mathrm{n}$ for univariate logistic models associating EUD with mean stool frequency $>1$. (D) LLH as a function of $\mathrm{n}$ for univariate logistic models associating EUD with grade $\geq 2$ late rectal pain.

Figure 3: probability of mean stool frequency $>1$ as a function of equivalent uniform dose, calculated with $\mathrm{n}=1$, and of presence of cardiovascular diseases. Continuous lines represent model curves, while symbols corresponds to toxicity rates observed in the pooled population (error bars represent standard deviations).

Figure 4: probability of grade $\geq 2$ late rectal pain as a function of equivalent uniform dose, calculated with $\mathrm{n}=0.35$, and of presence of grade $\geq 2$ acute gastrointestinal toxicity. Continuous lines represent model curves, while symbols corresponds to toxicity rates observed in the TROG 03.04 RADAR population (error bars represent standard deviations). 
Table 1: Distribution of patient characteristics in the Airopros 0102 and TROG 03.04 Radar populations and in the merged population. Differences in the variable rates between the two populations (Airopros 0102 vs TROG 03.04 Radar) were evaluated through the z-test for proportions. P-values for this test are reported in the table. Statistically significant p-values $(<0.05)$ are in italics.

\begin{tabular}{|c|c|c|c|c|c|c|c|c|c|c|}
\hline & \multicolumn{3}{|c|}{$\begin{array}{c}\text { Airopros } 0102 \\
\text { population } \\
\text { (659 patients) }\end{array}$} & \multicolumn{3}{|c|}{$\begin{array}{c}\text { TROG 03.04 Radar } \\
\text { population } \\
\text { (677 patients) }\end{array}$} & \multicolumn{3}{|c|}{$\begin{array}{c}\text { Merged population } \\
\text { (1336 patients) }\end{array}$} & \\
\hline & No & Yes & Yes\% & No & Yes & Yes\% & No & Yes & Yes\% & p-value \\
\hline $\begin{array}{l}\text { acute grade2-3 gastrointestinal } \\
\text { toxicity }\end{array}$ & 515 & 144 & 21.8 & 610 & 58 & 8.5 & 1134 & 202 & 15.1 & $<0.001$ \\
\hline $\begin{array}{l}\text { use of neoadjuvant/adjuvant } \\
\text { hormone therapy }\end{array}$ & 146 & 513 & 77.8 & 0 & 677 & 100.0 & 146 & 1190 & 89.7 & $<0.001$ \\
\hline use of anticoagulants & 525 & 134 & 20.3 & 572 & 105 & 15.5 & 1097 & 239 & 17.9 & 0.06 \\
\hline use of antihypertensives & 349 & 310 & 47.0 & 407 & 270 & 39.8 & 756 & 580 & 43.4 & 0.02 \\
\hline cardiovascular diseases & 621 & 38 & 5.8 & 483 & 194 & 28.7 & 1104 & 232 & 17.4 & $<0.001$ \\
\hline diabetes & 615 & 44 & 6.7 & 629 & 48 & 7.1 & 1271 & 92 & 6.9 & 0.65 \\
\hline presence of haemorrhoids & 522 & 137 & 20.8 & 662 & 15 & 2.2 & 1184 & 152 & 11.7 & 0.001 \\
\hline previous abdominal surgery & 596 & 63 & 9.6 & 664 & 13 & 1.9 & 1260 & 76 & 5.7 & $<0.001$ \\
\hline irradiation of seminal vesicles & 160 & 499 & 75.7 & 0 & 677 & 100.0 & 160 & 1176 & 88.0 & $<0.001$ \\
\hline irradiation of pelvic nodes & 621 & 38 & 5.8 & 677 & 0 & 0.0 & 1298 & 38 & 2.8 & $<0.001$ \\
\hline
\end{tabular}




\section{Supplementary materials}

\section{S1. Results for univariable logistic regression}

Univariate logistic regression results for mean stool frequency (Table S1) and grade $\geq 2$ rectal pain (Table $\mathrm{S} 2$ )

$\underline{\text { Table S1: Univariate logistic regression results for mean stool frequency }}$

\begin{tabular}{|c|c|c|c|c|c|}
\hline Variable & Coeff. & Std. Err. & $\begin{array}{l}z- \\
\text { score }\end{array}$ & $\mathbf{P}>|\mathbf{z}|$ & $\begin{array}{l}\text { Odds } \\
\text { Ratio }\end{array}$ \\
\hline EUD(EQD003) & -0.02 & 0.04 & -0.50 & 0.62 & 0.98 \\
\hline EUD(EQD005) & -0.01 & 0.04 & -0.23 & 0.82 & 0.99 \\
\hline EUD(EQD006) & 0.00 & 0.04 & -0.11 & 0.91 & 1.00 \\
\hline EUD(EQD008) & 0.00 & 0.04 & 0.09 & 0.93 & 1.00 \\
\hline EUD(EQD009) & 0.01 & 0.04 & 0.18 & 0.86 & 1.01 \\
\hline EUD(EQD015) & 0.02 & 0.04 & 0.60 & 0.55 & 1.02 \\
\hline EUD(EQD018) & 0.03 & 0.03 & 0.77 & 0.44 & 1.03 \\
\hline EUD(EQD024) & 0.03 & 0.03 & 1.03 & 0.30 & 1.03 \\
\hline EUD(EQD030) & 0.03 & 0.03 & 1.21 & 0.23 & 1.04 \\
\hline EUD(EQD035) & 0.04 & 0.03 & 1.32 & 0.19 & 1.04 \\
\hline EUD(EQD040) & 0.04 & 0.03 & 1.41 & 0.16 & 1.04 \\
\hline EUD(EQD045) & 0.04 & 0.02 & 1.47 & 0.14 & 1.04 \\
\hline EUD(EQD050) & 0.04 & 0.02 & 1.52 & 0.13 & 1.04 \\
\hline EUD(EQD055) & 0.04 & 0.02 & 1.56 & 0.12 & 1.04 \\
\hline EUD(EQD060) & 0.04 & 0.02 & 1.60 & 0.11 & 1.04 \\
\hline EUD(EQD065) & 0.04 & 0.02 & 1.62 & 0.11 & 1.04 \\
\hline EUD(EQD070) & 0.04 & 0.02 & 1.64 & 0.10 & 1.04 \\
\hline EUD(EQD075) & 0.04 & 0.02 & 1.66 & 0.10 & 1.04 \\
\hline EUD(EQD080) & 0.03 & 0.02 & 1.67 & 0.09 & 1.04 \\
\hline EUD(EQD085) & 0.03 & 0.02 & 1.68 & 0.09 & 1.04 \\
\hline EUD(EQD090) & 0.03 & 0.02 & 1.69 & 0.09 & 1.03 \\
\hline EUD(EQD095) & 0.03 & 0.02 & 1.70 & 0.09 & 1.03 \\
\hline Mean EQD2Gy & 0.03 & 0.02 & 1.71 & 0.09 & 1.03 \\
\hline V20Gy & 0.02 & 0.01 & 1.12 & 0.26 & 1.02 \\
\hline V25Gy & 0.01 & 0.01 & 1.07 & 0.29 & 1.01 \\
\hline V30Gy & 0.01 & 0.01 & 1.21 & 0.23 & 1.01 \\
\hline V35Gy & 0.01 & 0.01 & 1.43 & 0.15 & 1.01 \\
\hline V40Gy & 0.02 & 0.01 & 2.21 & 0.03 & 1.02 \\
\hline V45Gy & 0.01 & 0.01 & 1.10 & 0.27 & 1.01 \\
\hline V50Gy & 0.01 & 0.01 & 1.06 & 0.29 & 1.01 \\
\hline V55Gy & 0.01 & 0.01 & 0.99 & 0.32 & 1.01 \\
\hline
\end{tabular}




\begin{tabular}{|l|l|l|l|l|l|}
\hline V60Gy & 0.01 & 0.01 & 0.70 & 0.48 & 1.01 \\
\hline V65Gy & 0.00 & 0.01 & -0.36 & 0.72 & 1.00 \\
\hline V70Gy & -0.03 & 0.02 & -1.70 & 0.09 & 0.97 \\
\hline V75Gy & -0.05 & 0.05 & -1.00 & 0.32 & 0.95 \\
\hline Cardiovascular diseases & 0.55 & 0.34 & 1.62 & 0.11 & 1.73 \\
\hline Previous abdominal surgery & 0.36 & 0.61 & 0.59 & 0.56 & 1.44 \\
\hline Use of anticoagulants & 0.12 & 0.38 & 0.31 & 0.76 & 1.12 \\
\hline use of antihypertensives & -0.22 & 0.31 & -0.72 & 0.47 & 0.80 \\
\hline diabetes & 0.01 & 0.61 & 0.02 & 0.98 & 1.01 \\
\hline $\begin{array}{l}\text { Neoadjuvant/adjuvant } \\
\text { androgen deprivation }\end{array}$ & 0.64 & 0.73 & 0.87 & 0.38 & 1.89 \\
\hline Irradiation of pelvic nodes & 0.63 & 0.75 & 0.84 & 0.40 & 1.88 \\
\hline Irradiation of seminal vesicles & 0.97 & 0.73 & 1.33 & 0.18 & 2.65 \\
\hline $\begin{array}{l}\text { Presence of acute grade } \geq \mathbf{2} \\
\text { gastrointestinal toxicity }\end{array}$ & 0.94 & 0.34 & 2.75 & 0.01 & 2.55 \\
\hline
\end{tabular}

$\underline{\text { Table S2: Univariate logistic regression results for grade } \geq 2 \text { rectal pain }}$

\begin{tabular}{|l|l|l|l|l|l|}
\hline Variable & Coeff. & Std. Err. & $\begin{array}{l}\mathbf{Z}- \\
\text { score }\end{array}$ & $\mathbf{P}>|\mathbf{z}|$ & $\begin{array}{l}\text { Odds } \\
\text { Ratio }\end{array}$ \\
\hline Cardiovascular diseases & 0.43 & 0.46 & 0.93 & 0.35 & 1.53 \\
\hline Previous abdominal surgery & 1.00 & 1.07 & 0.94 & 0.35 & 2.72 \\
\hline Use of anticoagulants & 0.20 & 0.57 & 0.35 & 0.73 & 1.22 \\
\hline use of antihypertensives & 0.11 & 0.45 & 0.24 & 0.81 & 1.11 \\
\hline diabetes & 0.33 & 0.76 & 0.43 & 0.67 & 1.39 \\
\hline $\begin{array}{l}\text { Neoadjuvant/adjuvant } \\
\text { androgen deprivation }\end{array}$ & 1.55 & 0.50 & 3.08 & 0.00 & 4.72 \\
\hline V20Gy & 0.02 & 0.02 & 0.81 & 0.42 & 1.02 \\
\hline V25Gy & 0.02 & 0.02 & 0.99 & 0.32 & 1.02 \\
\hline V30Gy & 0.01 & 0.01 & 1.00 & 0.32 & 1.01 \\
\hline V35Gy & 0.02 & 0.01 & 1.35 & 0.18 & 1.02 \\
\hline V40Gy & 0.03 & 0.01 & 2.39 & 0.02 & 1.03 \\
\hline V45Gy & 0.02 & 0.01 & 1.75 & 0.08 & 1.02 \\
\hline V50Gy & 0.02 & 0.01 & 1.63 & 0.10 & 1.02 \\
\hline V55Gy & 0.02 & 0.02 & 1.41 & 0.16 & 1.02 \\
\hline V60Gy & 0.03 & 0.02 & 1.66 & 0.10 & 1.03 \\
\hline V65Gy & 0.01 & 0.02 & 0.72 & 0.47 & 1.01 \\
\hline V70Gy & 0.04 & 0.03 & 1.49 & 0.14 & 1.04 \\
\hline V75Gy & -0.18 & 0.68 & -0.27 & 0.79 & 0.83 \\
\hline EUD(EQD003) & 0.07 & 0.07 & 0.97 & 0.33 & 1.07 \\
\hline EUD(EQD005) & 0.09 & 0.07 & 1.23 & 0.22 & 1.09 \\
\hline
\end{tabular}




\begin{tabular}{|l|l|l|l|l|l|}
\hline EUD(EQD006) & 0.09 & 0.07 & 1.32 & 0.19 & 1.10 \\
\hline EUD(EQD008) & 0.10 & 0.07 & 1.46 & 0.14 & 1.10 \\
\hline EUD(EQD009) & 0.10 & 0.06 & 1.51 & 0.13 & 1.10 \\
\hline EUD(EQD015) & 0.10 & 0.06 & 1.71 & 0.09 & 1.10 \\
\hline EUD(EQD018) & 0.09 & 0.05 & 1.77 & 0.08 & 1.10 \\
\hline EUD(EQD024) & 0.09 & 0.05 & 1.84 & 0.07 & 1.09 \\
\hline EUD(EQD030) & 0.08 & 0.04 & 1.87 & 0.06 & 1.08 \\
\hline EUD(EQD035) & 0.08 & 0.04 & 1.87 & 0.06 & 1.08 \\
\hline EUD(EQD040) & 0.07 & 0.04 & 1.87 & 0.06 & 1.08 \\
\hline EUD(EQD045) & 0.07 & 0.04 & 1.86 & 0.06 & 1.07 \\
\hline EUD(EQD050) & 0.07 & 0.04 & 1.85 & 0.06 & 1.07 \\
\hline EUD(EQD055) & 0.06 & 0.04 & 1.83 & 0.07 & 1.07 \\
\hline EUD(EQD060) & 0.06 & 0.03 & 1.82 & 0.07 & 1.06 \\
\hline EUD(EQD065) & 0.06 & 0.03 & 1.80 & 0.07 & 1.06 \\
\hline EUD(EQD070) & 0.06 & 0.03 & 1.79 & 0.07 & 1.06 \\
\hline EUD(EQD075) & 0.06 & 0.03 & 1.77 & 0.08 & 1.06 \\
\hline EUD(EQD080) & 0.06 & 0.03 & 1.76 & 0.08 & 1.06 \\
\hline EUD(EQD085) & 0.06 & 0.03 & 1.75 & 0.08 & 1.06 \\
\hline EUD(EQD090) & 0.05 & 0.03 & 1.74 & 0.08 & 1.06 \\
\hline EUD(EQD095) & 0.05 & 0.03 & 1.73 & 0.08 & 1.05 \\
\hline Mean EQD2Gy & 0.05 & 0.03 & 1.72 & 0.09 & 1.05 \\
\hline
\end{tabular}




\section{S1. Calibration plots}

Figure S1: model for mean stool frequency endpoint: model including equivalent uniform dose calculated with $\mathrm{n}=1$ and cardiovascular diseases. The regression equation for fitted calibration plot is reported.

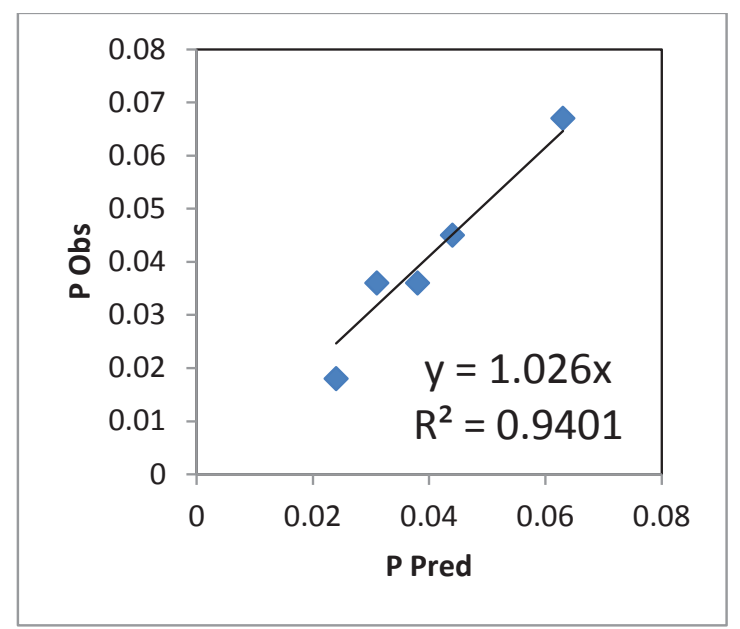

Figure S2: model for mean stool frequency endpoint: model including equivalent uniform dose calculated with $n=1$, cardiovascular diseases and presence of acute $\geq 2$ gastrointestinal toxicity. The regression equation for fitted calibration plot is reported.

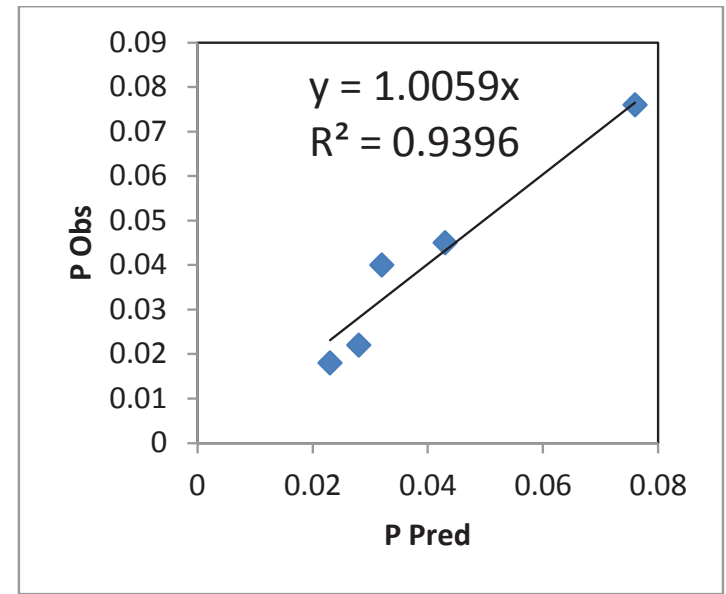


Figure S3: model for late grade $\geq 2$ rectal pain endpoint: model including equivalent uniform dose calculated with $\mathrm{n}=0.35$. The regression equation for fitted calibration plot is reported.

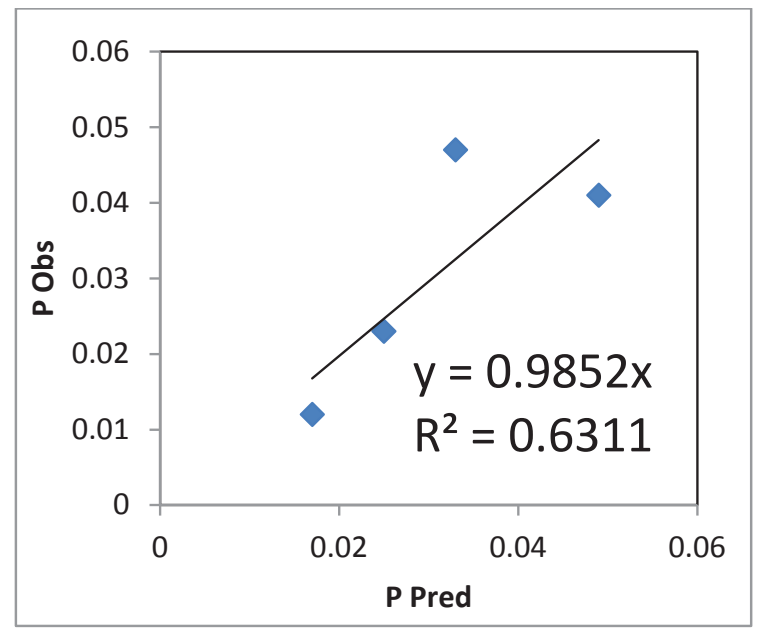

Figure S4: model for for late grade $\geq 2$ rectal pain endpoint: model including equivalent uniform dose calculated with $\mathrm{n}=0.35$ and presence of acute $\geq 2$ gastrointestinal toxicity. The regression equation for fitted calibration plot is reported.

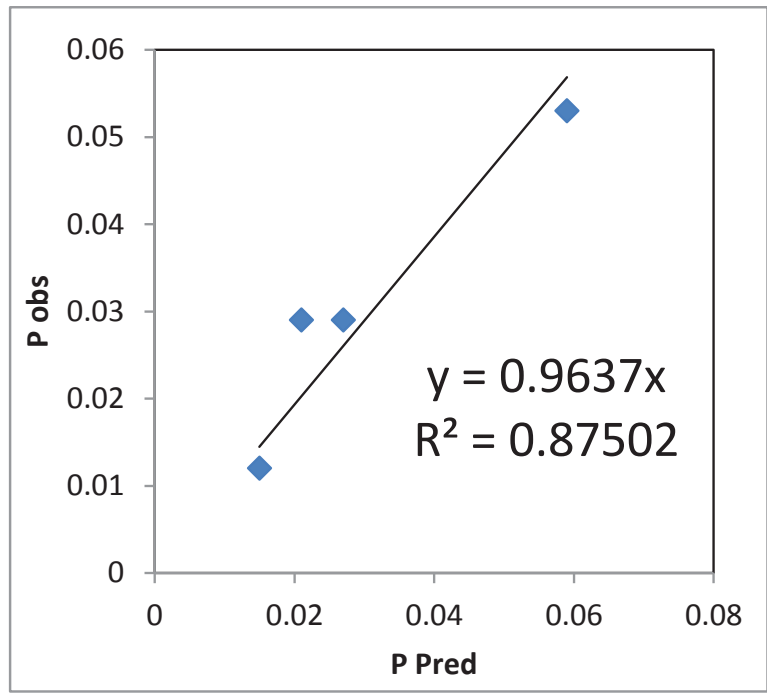



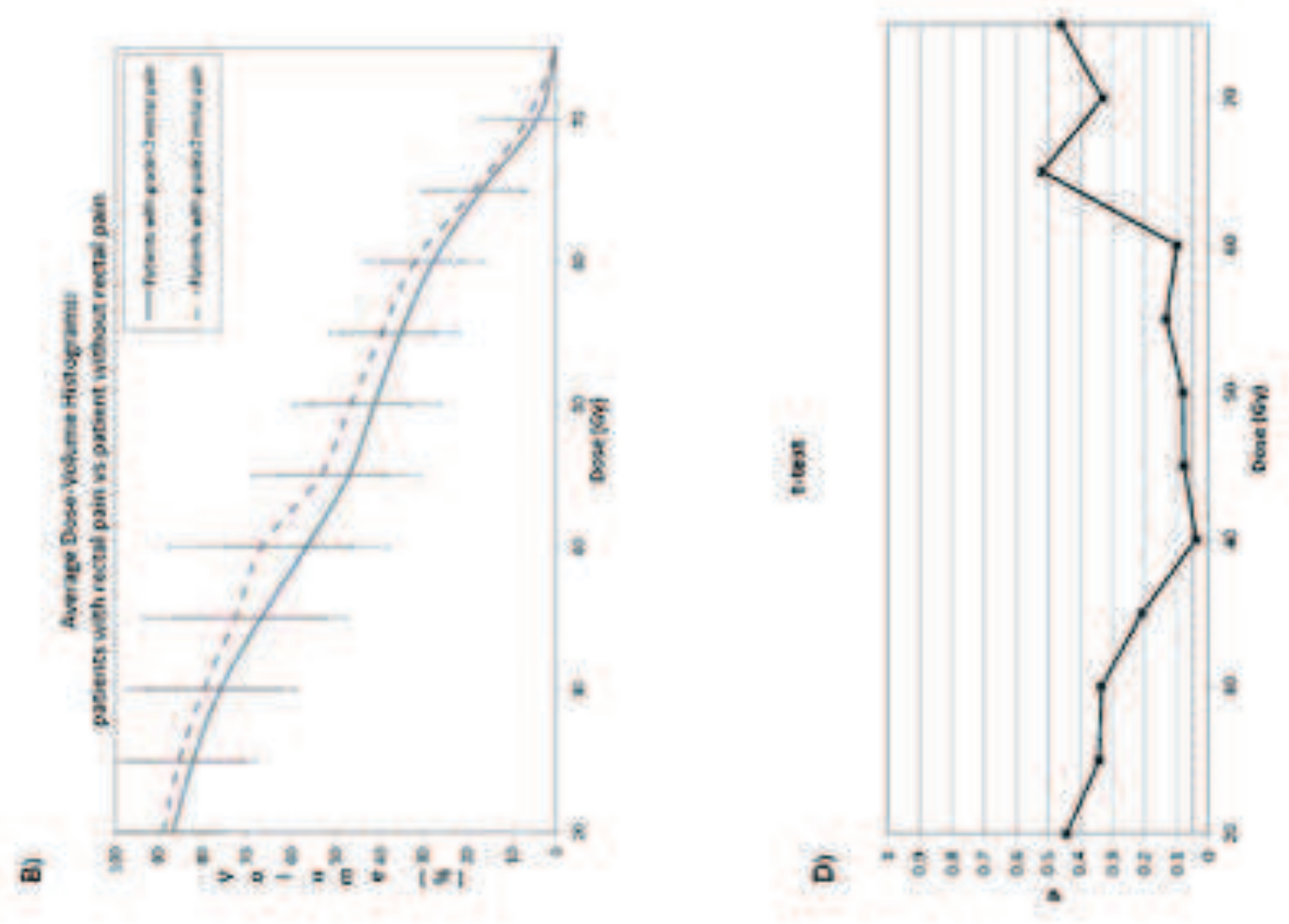

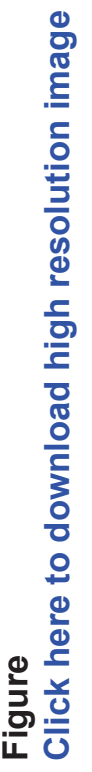
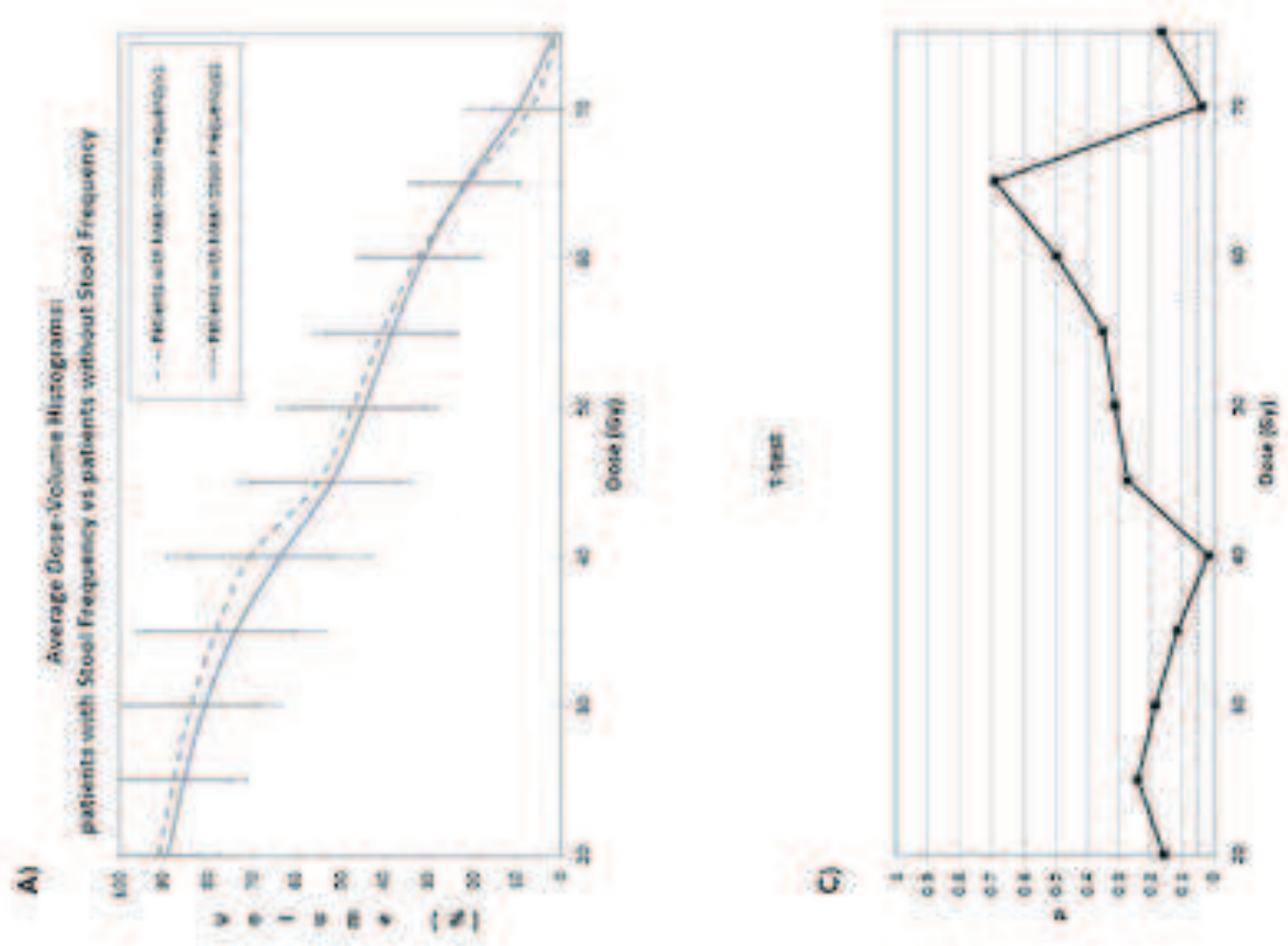

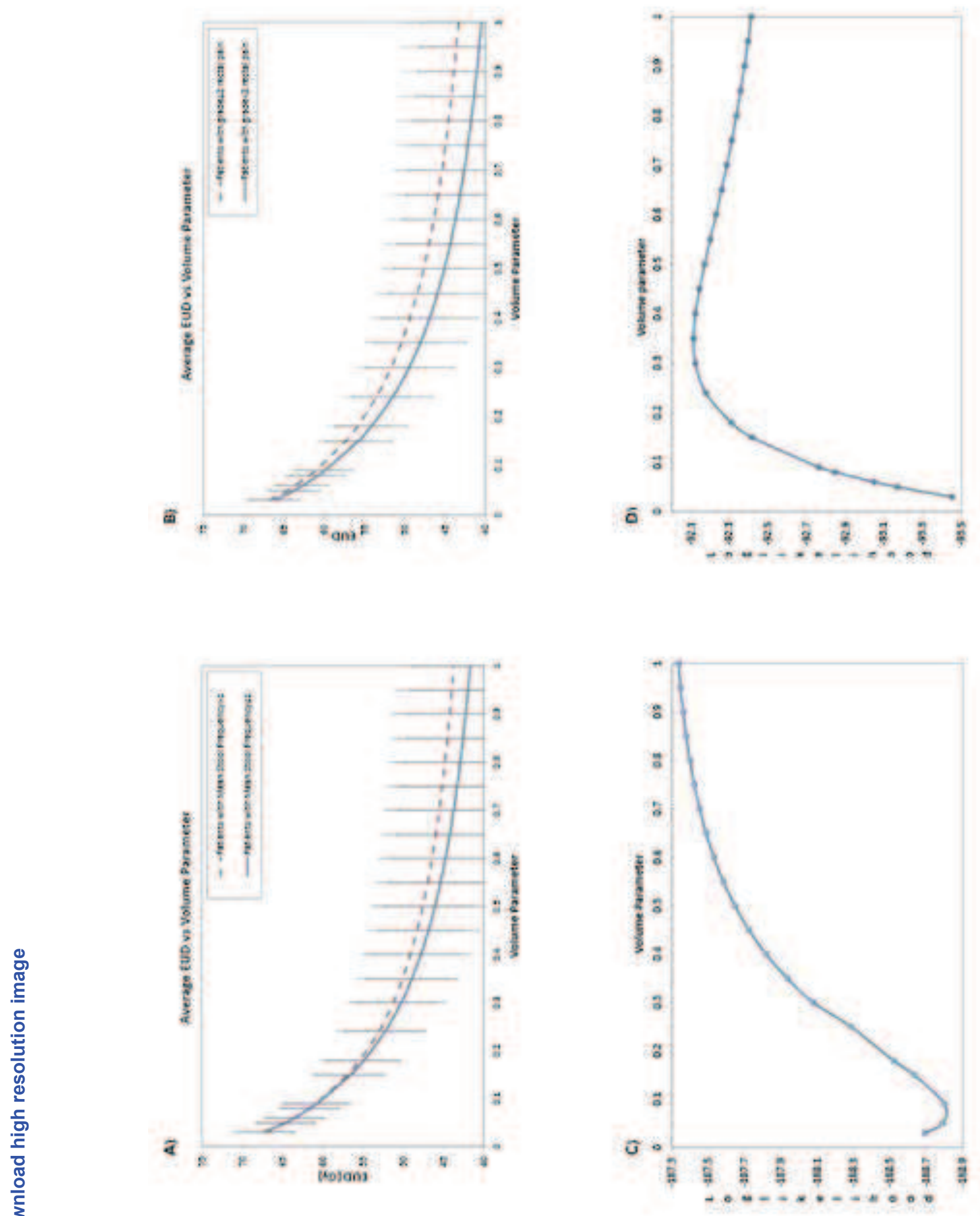


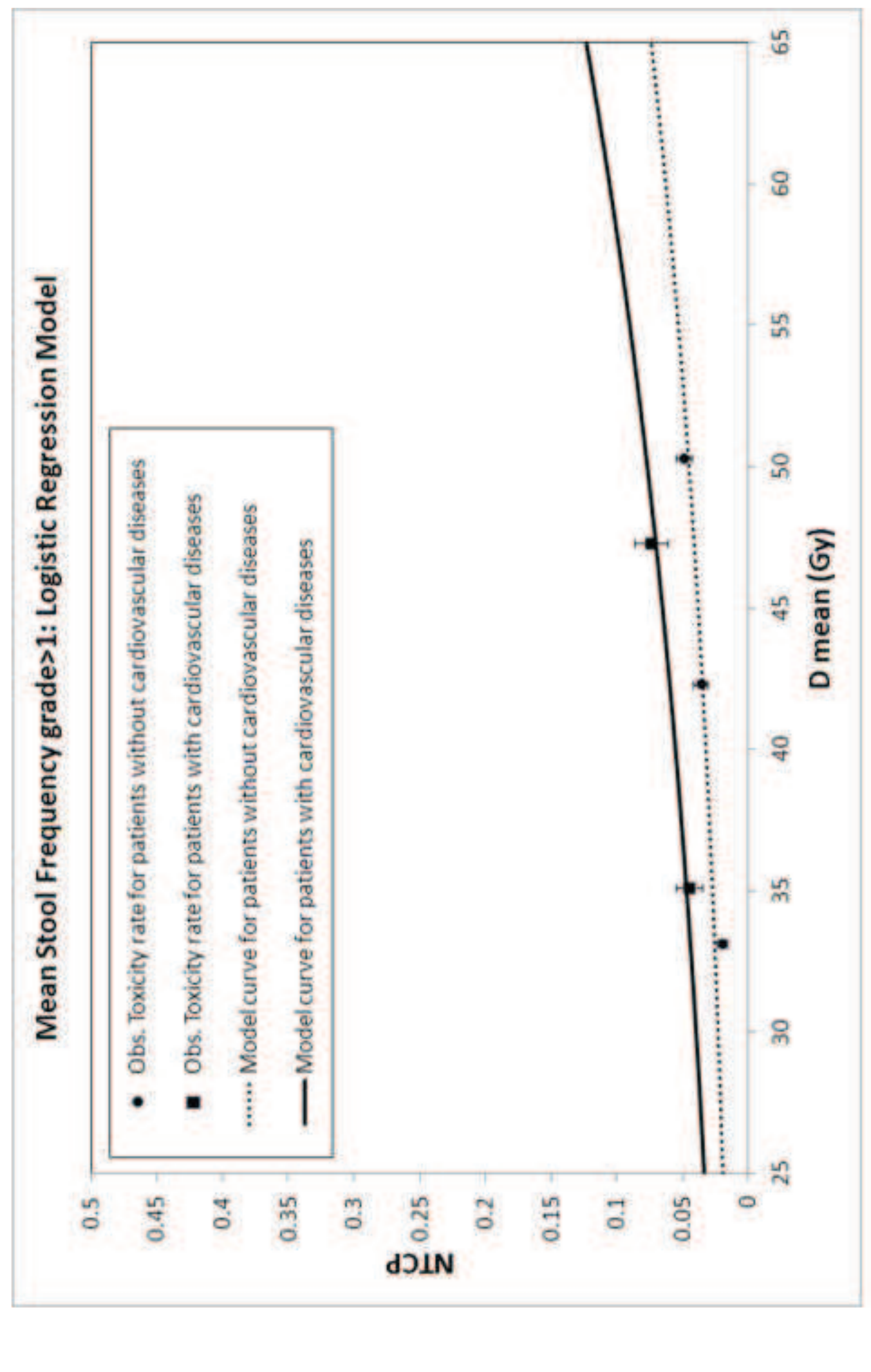




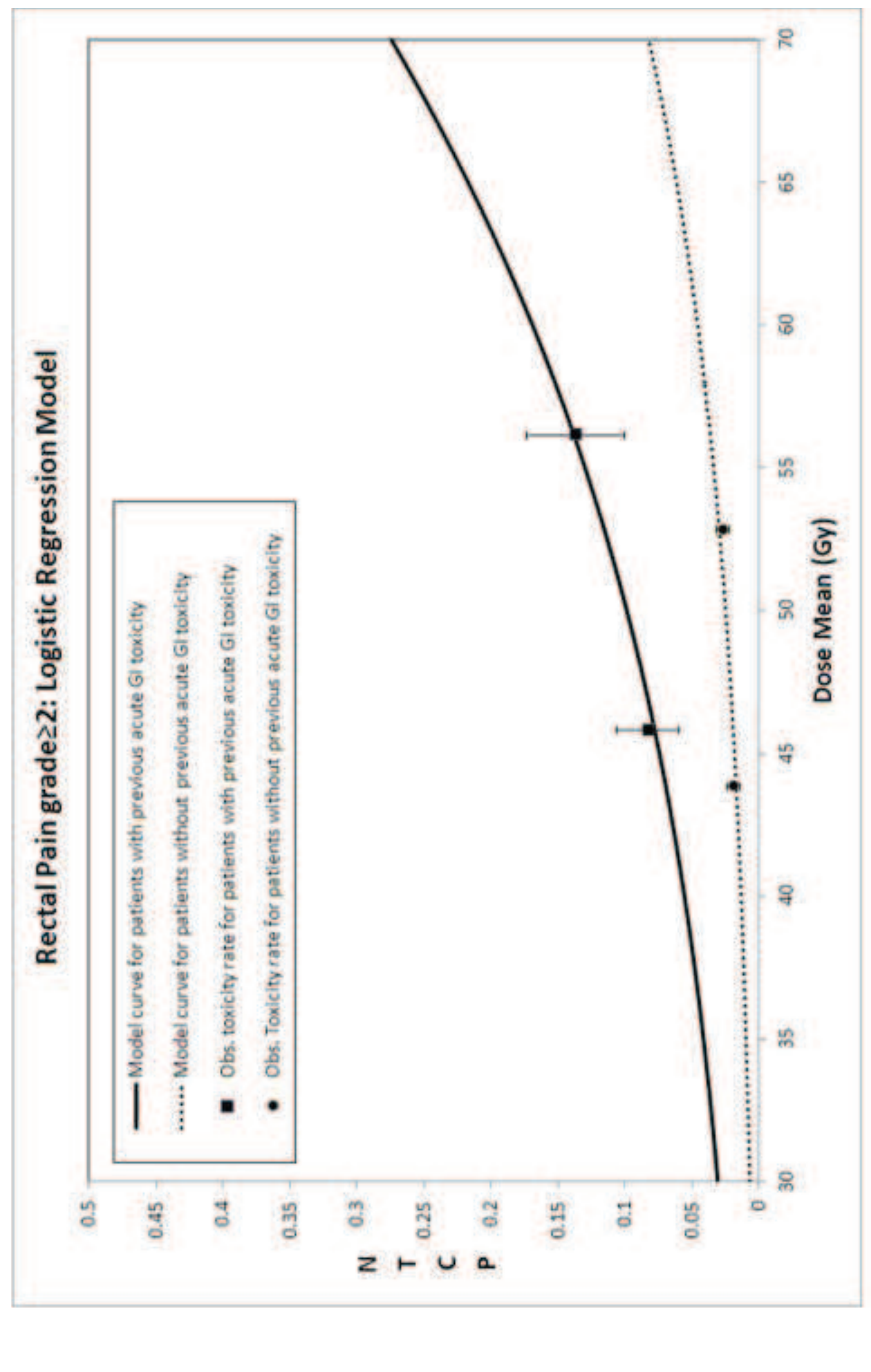

